\title{
THE ASYMPTOTIC BEHAVIOR NEAR THE CREST OF WAVES OF EXTREME FORM
}

\author{
J. B. MCLEOD
}

\begin{abstract}
The angle which the free boundary of an extreme wave makes with the horizontal is the solution of a singular, nonlinear integral equation. It has been proved only recently that solutions exist and that (as Stokes suggested in 1880) these solutions represent waves with sharp crests of included angle $\frac{2}{3} \pi$. Amick and Fraenkel have investigated the asymptotic behavior of the free surface near the crest and obtained an asymptotic expansion for this behavior, but are unable to say whether the leading term in this expansion has a nonzero coefficient (and so whether it is in fact the leading term or not). The present paper shows that the coefficient is nonzero and determines its sign.
\end{abstract}

1. Introduction. This note is in the nature of a postscript to the paper [1], Behavior near the crest of waves of extreme form, by Amick and Fraenkel. In it they discuss gravity waves, of permanent and extreme form, on the free surface of an ideal liquid, the flow being two-dimensional, irrotational and in a vertical plane. By a wave of extreme form is meant the "largest" member of a one-parameter family of such waves, and it is characterized by a sharp crest of included angle $\frac{2}{3} \pi$. The existence of such waves was conjectured by Stokes in 1880 and recently proved by Amick, Fraenkel, and Toland [2], where a fuller account of the problem is given. (See also [3] for background information.)

Amick and Fraenkel are concerned with the asymptotic behavior of the free surface of the wave near the crest. As is described in [1], the notation of which we adopt, it is a matter of discussing the behavior as $\zeta \downarrow 0$ of a solution

$$
\psi(\zeta)=\frac{1}{3 \pi} \int_{0}^{\infty} \frac{\omega(\eta) \sin \psi(\eta)}{\int_{0}^{\eta} \omega(\tau) \sin \psi(\tau) d \tau} \log \left|\frac{\zeta+\eta}{\zeta-\eta}\right| d \eta,
$$

where $0<\zeta<\infty$ and

$$
\omega(\eta)=\left(1+\eta^{2}\right)^{-1 / 2}\left(1+b \eta^{2}\right)^{-1 / 2}
$$

the constant $b$ satisfying $0 \leqslant b \leqslant 1$. (The value $b=0$ corresponds to solitary waves, the values $0<b<1$ to periodic waves of finite depth, and the value $b=1$ to periodic waves of infinite depth.) By a solution of (1.1) is meant a function $\psi$ satisfying (1.1) pointwise and such that $0<\psi<\frac{\pi}{2}, \psi$ is continuous on $(0, \infty)$,

Received by the editors October 21, 1985.

1980 Mathematics Subject Classification (1985 Revision). Primary 76B15, 45G05.

Key words and phrases. Water waves, nonlinear integral equations, asymptotic analysis. Sponsored by the United States Army under Contract No. DAAG29-80-C0041.

(C1987 American Mathematical Society $0002-9947 / 87 \$ 1.00+\$ .25$ per page 
$\psi(\zeta)=O\left(\zeta^{-1}\right)$ as $\zeta \rightarrow \infty$ and $\psi(\zeta) \rightarrow \frac{\pi}{6}$ as $\zeta \downarrow 0$. The existence of such solutions is known (see [1] for references).

It is standard that

$$
\frac{1}{3 \pi} \int_{0}^{\infty} \frac{1}{\eta} \log \left|\frac{\zeta+\eta}{\zeta-\eta}\right| d \eta=\frac{\pi}{6}
$$

and so (1.1) and (1.2) together yield

$$
\psi(\zeta)-\frac{1}{6} \pi=\frac{1}{3 \pi} \int_{0}^{\infty}\left(\frac{\omega(\eta) \sin \psi(\eta)}{\int_{0}^{\eta} \omega(\tau) \sin \psi(\tau) d \tau}-\frac{1}{\eta}\right) \log \left|\frac{\zeta+\eta}{\zeta-\eta}\right| d \eta
$$

A basic result of [1] is that

$$
\zeta^{-\beta_{1}}\left(\psi(\zeta)-\frac{\pi}{6}\right) \text { has a limit as } \zeta \downarrow 0,
$$

where $\beta_{1}$ is the smallest positive root of the equation

$$
\sqrt{3}(1+\beta)=\tan \frac{\pi \beta}{2}
$$

so that $0<\beta_{1}<1$. The authors go on to derive an asymptotic expansion for which (1.4) should provide the leading term, but it does remain possible in [1] that the limit in (1.4) is zero and that $\psi(\zeta)-\frac{\pi}{6}$ is of smaller order than $\zeta^{\beta_{1}}$. It is the object of the present note to show that this is not so, and that

$$
\psi(\zeta)-\frac{\pi}{6} \sim-A \zeta^{\beta_{1}} \text { as } \zeta \downarrow 0,
$$

where $A$ is a strictly positive constant. The method as in [1] is to use the Mellin transform on equation (1.3). The final result is therefore the following:

THEOREM. If $\psi$ is a solution of (1.1) in the sense described, then (1.6) holds.

2. Proof of the Theorem. We define the Mellin transform $\hat{f}$ of a function $f$ in the usual way by setting

$$
\hat{f}(s)=\int_{0}^{\infty} \zeta^{s-1} f(\zeta) d \zeta
$$

Since from [1] we know that (1.4) holds, the transform $\left(\overline{\psi-\frac{\pi}{6}}\right)(s)$ exists and represents an analytic function of $s$ for $-\beta_{1}<\operatorname{re}(s)<0$. For such $s$, we may multiply (1.3) by $\zeta^{s-1}$ and integrate to obtain

$$
\begin{gathered}
\left(\overline{\psi-\frac{\pi}{6}}\right)(s)=\frac{1}{3 \pi} \int_{0}^{\infty}\left(\frac{\omega(\eta) \sin \psi(\eta)}{\int_{0}^{\eta} \omega(\tau) \sin \psi(\tau) d \tau}-\frac{1}{\eta}\right)\left(\int_{0}^{\infty} \zeta^{s-1} \log \left|\frac{\zeta+\eta}{\zeta-\eta}\right| d \zeta\right) d \eta \\
=\frac{1}{3 \pi}\left(\int_{0}^{\infty} x^{s-1} \log \left|\frac{1+x}{1-x}\right| d x\right) \int_{0}^{\infty} \eta^{s}\left(\frac{\omega(\eta) \sin \psi(\eta)}{\int_{0}^{\eta} \omega(\tau) \sin \psi(\tau) d \tau}-\frac{1}{\eta}\right) d \eta
\end{gathered}
$$

by setting $\zeta=\eta x$. The interchange of order of integration is justified since both integrals in (2.1) are absolutely convergent. (Note that, by the properties of $\psi$, the $\eta$-integrand is $O\left(\eta^{s-1}\right)$ as $\eta \rightarrow \infty$ and $O\left(\eta^{s+\beta_{1}-1}\right)$ as $\eta \downarrow 0$.) If we now use the 
standard result that the first integral in (2.1) is just $\pi \tan \frac{1}{2} \pi s / s$, and integrate the second integral by parts, we obtain, for $-\beta_{1}<\operatorname{re}(s)<0$,

$$
\begin{gathered}
\left(\overline{\psi-} \frac{1}{6} \pi\right)(s)=-\frac{1}{3} \tan \frac{1}{2} \pi s \int_{0}^{\infty} \eta^{s-1} \log \left(\frac{2}{\eta} \int_{0}^{\eta} \omega(\tau) \sin \psi(\tau) d \tau\right) d \eta \\
=-\frac{1}{3} \tan \frac{1}{2} \pi s \int_{0}^{\infty} \eta^{s-1} \log \left(\frac{2}{\eta} \int_{0}^{\eta} \sin \psi(\tau) d \tau\right) d v-F(s)
\end{gathered}
$$

where

$$
F(s)=\frac{1}{3} \tan \frac{1}{2} \pi s \int_{0}^{\infty} \eta^{s-1} \log \left(\int_{0}^{\eta} \omega(\tau) \sin \psi(\tau) d \tau / \int_{0}^{\eta} \sin \psi(\tau) d \tau\right) d \eta
$$

Since $\omega(\tau)=1+O\left(\tau^{2}\right)$ as $\tau \downarrow 0$, we see that $F(s)$ is an analytic function of $s$ for $-1<\operatorname{re}(s)<0$. But also, since $0<\psi<\frac{\pi}{2}$ and $0<\omega \leqslant 1$, we see that $F(s)$ is strictly positive for $-1<s<0$. If we further write the logarithm in (2.3) in the form

$$
\log \left(1+\frac{2}{\eta} \int_{0}^{\eta}\left(\sin \psi(\tau)-\frac{1}{2}\right) d \tau\right.
$$

and note that $\log (1+t) \leqslant t$ for $t>-1$ and $\sin \psi-\frac{1}{2} \leqslant \frac{1}{2} \sqrt{3}\left(\psi-\frac{\pi}{6}\right)$ for $0<\psi<\frac{\pi}{2}$, we see that $(2.3)$ becomes

$$
\left(\overline{\psi-\frac{\pi}{6}}\right)(s)=-\frac{1}{\sqrt{3}} \tan \frac{1}{2} \pi s \int_{0}^{\infty} \eta^{s-2}\left(\int_{0}^{\eta}\left(\psi(\tau)-\frac{\pi}{6}\right) d \tau\right) d \eta-F_{1}(s),
$$

where $F_{1}(s)$ is a function analytic for $\max \left(-1,-2 \beta_{1}\right)<\operatorname{re}(s)<0$ and strictly positive for $\max \left(-1,-2 \beta_{1}\right)<s<0$. (In fact, $\beta_{1}$ can be explicitly evaluated from (1.5), and $2 \beta_{1}>1$.)

If finally we perform an integration by parts on the integral in (2.5), we obtain, for $-\beta_{1}<\operatorname{re}(s)<0$,

$$
\left(1-\frac{1}{\sqrt{3}} \frac{\tan \frac{1}{2} \pi s}{s-1}\right)\left(\overline{\psi-\frac{\pi}{6}}\right)(s)=-F_{1}(s),
$$

or

$$
\left(\overline{\psi-\frac{\pi}{6}}\right)(s)=-\frac{\sqrt{3}(1-s) F_{1}(s)}{\sqrt{3}(1-s)+\tan \frac{1}{2} \pi s},
$$

and we use the inversion theorem on this. Thus

$$
\psi(\zeta)-\frac{\pi}{6}=\lim _{T \rightarrow \infty} \frac{1}{2 \pi i} \int_{c-i T}^{c+i T} \frac{-\sqrt{3}(1-s) F_{1}(s)}{\sqrt{3}(1-s)+\tan \frac{1}{2} \pi s} \zeta^{-s} d s,
$$

where $c$ is any number satisfying $-\beta_{1}<c<0$.

Now move the path of integration from $\operatorname{re}(s)=c$ to $\operatorname{re}(s)=-l$, where $\beta_{1}<l<1$. Since the integrand is analytic for $-1<\operatorname{re}(s)<0$ except for a simple pole at $s=-\beta_{1}$, where the residue is

$$
-\frac{\sqrt{3}\left(1+\beta_{1}\right) F_{1}\left(\beta_{1}\right)}{-\sqrt{3}+\frac{1}{2} \pi \sec ^{2} \frac{1}{2} \pi \beta_{1}} \zeta^{\beta_{1}}=-A \zeta^{\beta_{1}},
$$


say, where $A>0$, we have

$$
\left(\psi-\frac{\pi}{6}\right)(s)=-A \zeta^{\beta_{1}}+\lim _{T \rightarrow \infty} \frac{1}{2 \pi i} \int_{-l-i T}^{-l+i T} \frac{-\sqrt{3}(1-s) F_{1}(s)}{\sqrt{3}(1-s)+\tan \frac{1}{2} \pi s} \zeta^{-s} d s
$$

The proof of the theorem is thus complete provided we can show

(i) that the contribution from the horizontal portions of the transformed contour, on $\operatorname{im}(s)= \pm T$, are negligible as $T \rightarrow \infty$, and

(ii) that the remaining integral on the right of $(2.6)$ is $O\left(\zeta^{l}\right)$ as $\zeta \downarrow 0$.

To prove (i), consider $F(s)$ as given by (2.4). Since the logarithm is $O\left(\eta^{2}\right)$ as $\eta \downarrow 0$ and $O(\log \eta)$ as $\eta \rightarrow \infty$, we see that $|F(s)| \rightarrow 0$ as $\operatorname{im}(s) \rightarrow \pm \infty$, by the RiemannLebesgue lemma in its Mellin form, provided that $-1<\operatorname{re}(s)<0$. A similar remark can be made about $F_{1}(s)$, and this is sufficient to prove (i).

To prove (ii), we can integrate $(2.4)$ by parts to obtain

$$
F(s)=-\frac{1}{3 s} \tan \frac{1}{2} \pi s \int_{0}^{\infty} \eta^{s}\left(\frac{w(\eta) \sin \psi(\eta)}{\int_{0}^{\eta} w(\tau) \sin \psi(\tau) d \tau}-\frac{\sin \psi(\eta)}{\int_{0}^{\eta} \sin \psi(\tau) d \tau}\right) d \eta .
$$

With $\operatorname{re}(s)=-l$, consider $F(s)$ as a function of $\operatorname{im}(s)$. Since the integrand is $O\left(\eta^{s-1}\right)$ as $\eta \rightarrow \infty$ and $O\left(\eta^{s+1}\right)$ as $\eta \downarrow 0$, Parseval's theorem in its Mellin form tells us that the integral is $L^{2}(-\infty, \infty)$ as a function of $\mathrm{im}(s)$. In view of the factor $s^{-1}$ in front of the integral, we see that $F \in L(-\infty, \infty)$. Similar remarks can be made about $F_{1}$, and it is then clear that the integral in (2.6) is $O\left(\zeta^{l}\right)$.

This completes the proof of the theorem.

\section{REFERENCES}

1. C. J. Amick and L. E. Fraenkel, Behavior near the crest of waves of extreme form, Trans. Amer. Math. Soc. 299 (1987), 273-298.

2. C. J. Amick, L. E. Fraenkel and J. F. Toland, On the Stokes conjecture for the wave of extreme form, Acta Math. 148 (1982), 193-214.

3. L. M. Milne-Thomson, Theoretical hydrodynamics, 5th ed., Macmillan, 1968.

Department of Mathematics, Purdue University, West Lafayette, Indiana 47907

DePartment of Mathematics, OXFord University, OXFord, ENGLAND 